\title{
SOME RESULTS CONCERNING COMPLETELY 0-SIMPLE SEMIGROUPS
}

\author{
BY GÉRARD LALLEMENT AND MARIO PETRICH
}

Communicated by E. Pitcher, July 21, 1964

We follow the notation and terminology of [1]. A semigroup $T$ with zero is said to be 0-rectangular if it has the property: if all the products at the vertices of a closed polygonal line (with a finite number of vertices) of the multiplication table are all but one equal to a nonzero element $m$ and the remaining product is not zero, then it is also equal to $m$. A rectangular 0 -band is a Rees matrix semigroup with zero over the one-element group.

TheOREM 1. Let $S=\mathfrak{N}^{0}(G ; I, \Lambda ; P)$. Then the following statements are equivalent:

(a) $S \cong G \times E / G \times\{0\}$, where $E$ is a rectangular 0 -band;

(b) there exist invertible matrices $U(I \times I)$ and $V(\Lambda \times \Lambda)$ such that $Q=V P U$ is a regular matrix all of whose nonzero entries are equal to 1 ;

(c) there exist mappings $\alpha: I \rightarrow G, \beta: \Lambda \rightarrow G$ such that $p_{\lambda_{i}}=\beta(\lambda) \alpha(i)$ if $p_{\lambda_{i}} \neq 0$;

(d) $S$ is 0-rectangular;

(e) if $p_{\lambda_{1} i_{1}}, p_{\lambda_{1} i_{2}}, p_{\lambda_{2} i_{2}}, \cdots, p_{\lambda_{n} i_{n}}, p_{\lambda_{n} i_{1}} \neq 0$, then $p_{\lambda_{1} i_{1}}^{-1} p_{\lambda_{1} i_{2}} p_{\lambda_{2} i_{2}}^{-1} \cdots$ $p_{\lambda_{n} i_{n}}^{-1} p_{\lambda_{n} i_{1}}=1$

(f) $S$ has a subsemigroup intersecting each He-class of $S$ in exactly one element.

The semigroup in (f) need not be unique. We note that an analogous result is valid for completely simple semigroups (i.e., without zero); in such a case (b) and (c) remain essentially the same, (a) becomes $S \cong G \times E, E$ is a rectangular band, in (d) " 0 -rectangular" is replaced by "rectangular," in (e) it suffices to take four entries of $P$ at a time, and (f) states that idempotents form a semigroup (and thus in this case the semigroup in (f) is unique).

An ideal $I$ of a semigroup $T$ is said to be a matrix ideal of $T$ if: for all $a, b, c \in T$, (a) $a T b \subseteq I$ implies $a \in I$ or $b \in I$, (b) $a b c \in I$ implies $a b \in I$ or $b c \in I$.

TheOREM 2. Let $S$ be a semigroup with a completely 0-simple ideal $M$. In order that there exist an $M$-homomorphism of $S$ onto $M$, it is necessary and sufficient that (0) be a matrix ideal of $S$, and the restriction to $M$ of the finest congruence $\rho$ on $S$, having 0 as one of its classes and such that $S / \rho$ is a rectangular 0 -band, coincides with the $\mathcal{H}$-equivalence on $M$. 
A corresponding result is valid for semigroups with a completely simple ideal; in such a case $S / \rho$ in the theorem is a rectangular band (cf. [2]).

A semigroup $T$ with zero is said to be 0 -inversive if for every $a \in T$, $a \neq 0$, there exists $x \in T$ such that $a x$ is a nonzero idempotent. $T$ is weakly 0 -cancellable if $x a=x b \neq 0$ and $a y=b y \neq 0$ implies $a=b$ [3].

THEOREM 3. The following conditions on a semigroup $S$ with zero are equivalent:

(a) $S$ is regular and all its nonzero idempotents are primitive;

(b) $S$ is regular and for all $a, x \in S, a x a=a \neq 0$ implies $x a x=x$;

(c) (i) for all $a \in S$, there are $e, f \in S$ such that $a=e a=a f$,

(ii) to each $a \in S, a \neq 0$, and $e, f$ as in (i), corresponds a unique $a^{\prime} \in S$ such that $a a^{\prime}=e, a^{\prime} a=f$;

(d) $S$ is 0-inversive and weakly 0-cancellable;

(e) $S$ is 0-inversive and every nonzero principal left (and right) ideal of $S$ is 0-minimal;

(f) $S$ is a mutually annihilating sum [4] of completely 0-simple semigroups.

As a consequence of this theorem one obtains certain results concerning inverse semigroups with zero (cf. [5]). For in (a) and (b) one substitutes "regular" by "inverse" which is equivalent to uniqueness of $e$ and $f$ in (c), and in (f) "completely 0 -simple" is replaced by "Brandt semigroup"; the remaining items also have their analogues for this case.

\section{REFERENCES}

1. A. H. Clifford and G. B. Preston, The algebraic theory of semigroups, Math. Surveys No. 7, Amer. Math. Soc., Providence, R. I., 1961.

2. $\mathrm{H}$. Hashimoto, On the structure of semigroups containing minimal left ideals and minimal right ideals, Proc. Japan Acad. 31 (1955), 264-266.

3. G. Lallement, Sur les homomorphismes d'un demi-groupe sur un demi-groupe completement-0-simple, C. R. Acad. Sci. Paris 258 (1964), 3609-3612.

4. E. S. Ljapin, Normal complexes of associative systems, Izv. Akad. Nauk SSSR Ser. Mat. 14 (1950), 179-192. (Russian)

5. P. S. Venkatesan, On a class of inverse semigroups, Amer. J. Math. 84 (1962), 578-582.

University of Paris (C.N.R.S.) AND LEHIGH UNIVERSITY 